\title{
An Innovative Solutions for the Generalized FitzHugh-Nagumo Equation by Using the Generalized $\left(\frac{G^{\prime}}{G}\right)$-Expansion Method
}

\author{
Sayed Kahlil Elagan ${ }^{1,2}$, Mohamed Sayed ${ }^{2,3}$, Yaser Salah Hamed ${ }^{2,3}$ \\ ${ }^{1}$ Department of Mathematics, Faculty of Science, Menofia University, Menouf, Egypt \\ ${ }^{2}$ Department of Mathematics, Faculty of Science, Taif University, Taif, Kingdom of Saudi Arabia (KSA) \\ ${ }^{3}$ Department of Engineering Mathematics, Faculty of Electronic Engineering, Menofia University, Menouf, Egypt \\ E-mail: \{sayed_khalil2000, moh_6_11,eng_yaser_salah\}@yahoo.com \\ Received February 24, 2011; revised March 4, 2011; accepted March 8, 2011
}

\section{Abstract}

In this paper, the generalized $\left(\frac{G^{\prime}}{G}\right)$-expansion method is used for construct an innovative explicit traveling wave solutions involving parameter of the generalized FitzHugh-Nagumo equation $u_{t}=u_{x x}-u(1-u)(a(t)-u)$, for some special parameter $a(t)$ where $G=G(\xi)$ satisfies a second order linear differential equation $G^{\prime \prime}+\lambda G^{\prime}+\mu G=0, \quad \xi=p(t) x+q(t)$, where $p(t)$ and $q(t)$ are functions of $t$.

Keywords: FitzHugh-Nagumo Equation, Generalized $\left(\frac{G^{\prime}}{G}\right)$-Expansion Method, Traveling Wave Solutions

\section{Introduction}

Phenomena in physics and other fields are often described by nonlinear evolution equations (NLEEs). When we want to understand the physical mechanism of phenomena in nature, described by nonlinear evolution equations, exact solutions for the nonlinear evolution equations have to be explored. For example, the wave phenomena observed in fluid dynamics [1,2], plasma and elastic media [3,4] and optical fibers [5,6], etc. In the past several decades, many effective methods for obtaining exact solutions of NLEEs have been proposed, such as Hirota's bilinear method [7], Backlund transformation [8], Painlevé expansion [9], sine-cosine method [10], homogeneous balance method [11], homotopy perturbation method [12-14], variational iteration method [15-18], asymptotic methods [19], non-perturbative methods [20], Adomian decomposition method [21], tanhfunction method [22-26], algebraic method [27-30]. Jacobi elliptic function expansion method [31-33], F-expansion method [34-36] and auxiliary equation method [37-40]. Recently, Wang et al. [41] introduced a new direct method called the $\left(\frac{G^{\prime}}{G}\right)$-expansion method to look for travelling wave solutions of NLEEs. The $\left(\frac{G^{\prime}}{G}\right)$-expansion method is based on the assumptions that the travelling wave solutions can be expressed by a polynomial in $\left(\frac{G^{\prime}}{G}\right)$, and that $G=G(\xi)$ satisfies a second order linear ordinary differential equation (LODE):

$G^{\prime \prime}+\lambda G^{\prime}+\mu G=0$, where $G^{\prime}=\frac{\mathrm{d} G(\xi)}{\mathrm{d} \xi}, G^{\prime \prime}=\frac{\mathrm{d}^{2} G(\xi)}{\mathrm{d} \xi^{2}}$, $\xi=x-V t, V$ is a constant. The degree of the polynomial can be determined by considering the homogeneous balance between the highest order derivative and nonlinear terms appearing in the given NLEE. The coefficients of the polynomial can be obtained by solving a set of algebraic equations resulted from the process of 
using the method. By using the $\left(\frac{G^{\prime}}{G}\right)$-expansion method, Wang et al. [41] successfully obtained more travelling wave solutions of four NLEEs. Very recently, Zhang et al. [42] proposed a generalized $\left(\frac{G^{\prime}}{G}\right)$-expansion method [42] to improve the work made in [41]. The main purpose of this paper is to use generalized $\left(\frac{G^{\prime}}{G}\right)$-expansion method to solve the generalized FitzHugh-Nagumo equation. The performance of this method is reliable, simple and gives many new solutions, its also standard and computerizable method which enable us to solve complicated nonlinear evolution equations in mathematical physics. The paper is organized as follows. In section 2, we describe briefly the generalized $\left(\frac{G^{\prime}}{G}\right)$-expansion method, where $G=G(\xi)$ satisfies the second order linear ordinary differential equation $G^{\prime \prime}+\lambda G^{\prime}+\mu G=0$, $\xi=p(t) x+q(t)$ In section 3, we apply this method to the FitzHugh-Nagumo equation. In section 4, some conclusions are given.

\section{Description the Generalized}$$
\left(\frac{G^{\prime}}{G}\right) \text {-Expansion Method }
$$

Suppose that we have the following nonlinear partial differential equation

$$
P\left(u, u_{t}, u_{x}, u_{t t}, u_{x t}, u_{x x}, \cdots\right)=0,
$$

we suppose its solution can be expressed by a polynomial $\left(\frac{G^{\prime}}{G}\right)$ as follows:

$$
u(\xi)=\sum_{i=1}^{n} \alpha_{i}(t)\left(\frac{G^{\prime}}{G}\right)^{i}+\alpha_{0}(t), \quad \alpha_{j}(t) \neq 0,
$$

where $\alpha_{0}(t)$ and $\alpha_{j}(t)$ are functions of $t$ $(j=1,2, \cdots, n)$ and $\xi=\xi(x, t)$ is a function of $x, t$ to be determine later, $G=G(\xi)$ satisfies the second order linear ordinary differential equation

$$
G^{\prime \prime}(\xi)+\lambda G^{\prime}(\xi)+\mu G(\xi)=0,
$$

To determine $u$ explicitly we take the following four steps.

Step 1. Determine the integer $n$ by balancing the highest order nonlinear term(s) and the highest order partial derivative of $u$ in Equation (2.1).

Step 2. Substitute Equation (2.2) along with Equation (2.3) into Equation (2.1) and collect all terms with the same order of $\left(\frac{G^{\prime}}{G}\right)$ together, the left hand side of Equation (2.1) is converted into a polynomial in $\left(\frac{G^{\prime}}{G}\right)$. Then set each coefficient of this polynomial to zero to derive a set of over-determined partial differential equations for $\alpha_{0}(t), \alpha_{i}(t)$ and $\xi$.

Step 3. Solve the system of all equations obtained in step 2 for $\alpha_{0}(t), \alpha_{i}(t)$ and $\xi$ by use of Maple.

Step 4. Use the results obtained in above steps to derive a series of fundamental solutions of Equation (2.3) depending on $\left(\frac{G^{\prime}}{G}\right)$, since the solutions of this equation have been well known for us, then we can obtain exact solutions of Equation (2.1).

\section{The FitzHugh-Nagumo Equation}

In this section, we apply the generalized $\left(\frac{G^{\prime}}{G}\right)$-expansion method to solve the generalized FitzHugh-Nagumo equation, construct the traveling wave solutions for it as follows:

Let us first consider the generalized FitzHugh-Nagumo equation

$$
u_{t}=u_{x x}-u(1-u)(a(t)-u)
$$

where $a(t)$ is a function of $t$. In order to look for the traveling wave solutions of Equation (3.1) we suppose that

$$
u(x, t)=u(\xi), \xi=p(t) x+q(t)
$$

Suppose that the solution of Equation (3.1) can be expressed by a polynomial in $\left(\frac{G^{\prime}}{G}\right)$ as follows

$$
u(\xi)=\sum_{i=1}^{n} \alpha_{i}(t)\left(\frac{G^{\prime}}{G}\right)^{i}+\alpha_{0}(t)
$$

Considering the homogeneous balance between $u_{x x}$ and $u^{3}$ in Equation (3.1) we required that $n+2=3 n$, then $n=1$. So we can write Equation (3.3) as

$$
u(\xi)=\alpha(t)\left(\frac{G^{\prime}}{G}\right)+\alpha_{0}(t) .
$$

Substituting Equation (3.4) into Equation (3.1) along with Equation (2.3). We obtain the following equations by comparing coefficients of $\left(\frac{G^{\prime}}{G}\right)$. When $j=3$ then

$$
0=2 \alpha_{1} p^{2}-\alpha_{1}^{3}
$$


We solve the equation by setting $\alpha_{1}=\sqrt{2} p$ (we could also set $\alpha_{1}=-\sqrt{2} p$ ). The equation for $j=2$ is

$$
-\alpha_{1}\left(p^{\prime} x+q^{\prime}\right)=3 \alpha_{1} p^{2} \lambda-3 \alpha_{0} \alpha_{1}^{2}+\alpha_{1}^{2}+\alpha_{1}^{2} a .
$$

We see from this equation that $p(t)$ must be a constant and then $\alpha_{1}(t)$ is also constant. Therefore, equation Equation (3.6) simplifies to

$$
-\alpha_{1} q^{\prime}=3 \alpha_{1} p^{2} \lambda-3 \alpha_{0} \alpha_{1}^{2}+\alpha_{1}^{2}+\alpha_{1}^{2} a .
$$

The equation for $j=1$ is

$$
\begin{aligned}
& -\alpha_{1} q^{\prime} \lambda=2 \alpha_{1} p^{2} \mu+\alpha_{1} p^{2} \lambda^{2}+2 \alpha_{0} \alpha_{1} \\
& -3 \alpha_{0}^{2} \alpha_{1}+2 \alpha_{0} \alpha_{1} a-\alpha_{1} a .
\end{aligned}
$$

We substitute Equation (3.7) into Equation (3.8) and obtain (after dividing by $\alpha_{1}$ )

$$
\begin{aligned}
& -3 \alpha_{1} \alpha_{0} \lambda+\alpha_{1} \lambda+\alpha_{1} a \lambda+2 p^{2} \lambda^{2}-2 \alpha_{0}+3 \alpha_{0}^{2} \\
& -2 \alpha_{0} a-2 p^{2} \mu+a=0 .
\end{aligned}
$$

We solve this equation for $a$ and obtain

$a(t)=\frac{3 \alpha_{1} \alpha_{0} \lambda-\alpha_{1} \lambda-2 p^{2} \lambda^{2}+2 \alpha_{0}-3 \alpha_{0}^{2}+2 p^{2} \mu}{\alpha_{1} \lambda-2 \alpha_{0}+1}$.

The equation for $j=0$ is

$$
\alpha_{0}^{\prime}-\alpha_{1} q^{\prime} \mu=\alpha_{1} p^{2} \lambda \mu-\alpha_{0} a+\alpha_{0}^{2}+\alpha_{0}^{2} a-\alpha_{0}^{3} .
$$

If we substitute Equation (3.7) and Equation (3.10) into Equation (3.11) we obtain

$$
\begin{aligned}
& \alpha_{0}^{\prime}\left(\alpha_{1} \lambda-2 \alpha_{0}+1\right)+\alpha_{0}^{4}-\alpha_{0}^{3}\left(2 \alpha_{1} \lambda+2\right) \\
& +\alpha_{0}^{2}\left(3 \alpha_{1}^{2} \mu+3 \alpha_{1} \lambda+1+2 p^{2} \lambda^{2}-2 p^{2} \mu\right) \\
& +\alpha_{0}\left(-3 \alpha_{1}^{2} \mu-4 \alpha_{1} p^{2} \lambda \mu-2 p^{2} \lambda^{2}+2 p^{2} \mu-\alpha_{1} \lambda\right) \\
& +2 \alpha_{1}^{2} \mu^{2} p^{2}+\alpha_{1}^{2} \mu+2 \alpha_{1} p^{2} \lambda \mu=0
\end{aligned}
$$

Now Equation (3.12) is an ordinary differential equation for $\alpha_{0}$. Therefore, $\alpha_{0}$ must have a special form in order to be a solution of this equation which means that the function $a(t)$ expressed in terms of $\alpha_{0}(t)$ by Equation (3.10) must also of a special form. This shows that we cannot solve all the equations if $a(t)$ is an arbitrary function.

We can still try to find solutions for some special $a(t)$. For example, we choose

$$
p=\frac{1}{\sqrt{2}}, \lambda=1, \mu=0 .
$$

Then $\alpha_{1}=1$ and Equation (3.12) simplifies to

$$
\alpha_{0}^{\prime}+\frac{3}{2} \alpha_{0}^{2}-\alpha_{0}-\frac{1}{2} \alpha_{0}^{3}=0 .
$$

One solution is

$$
\alpha_{0}(t)=1+\frac{1}{\sqrt{1+e^{t}}} .
$$

We find $a(t)$ from Equation (3.7) as

$$
\begin{gathered}
a(t)=\frac{1}{2}+\frac{3}{2} \sqrt{1+e^{t}} \\
q(t)=-3 \arctan h\left(\sqrt{1+e^{t}}\right) .
\end{gathered}
$$

We choose

$$
G(\xi)=1+e^{-\xi} .
$$

Then

$$
u=1+\frac{1}{\sqrt{1+e^{t}}}-\frac{e^{-\xi}}{1+e^{-\xi}}
$$

with

$$
\xi=\frac{x}{\sqrt{2}}-3 \arctan h\left(\sqrt{1+e^{t}}\right)
$$

is a solution of equation Equation (3.1) when $a(t)$ is given by Equation (3.13). Once can check with the computer that $u$ given by Equation (3.14) is really a solution of Equation (3.1). It is shows that this method is powerful in constructing exact solutions of NLEEs.

\section{Conclusions}

This study shows that the generalized $\left(\frac{G^{\prime}}{G}\right)$-expansion method is quite efficient and practically will suited for use in finding exact solutions for the problem considered here. New and more general excat solutions with arbitrary function $a(t)$ of the generalized FitzHughNagumo equation are obtained, from which some exponential function solutions are also derived when setting the arbitrary function as special values. We construct an innovative explicit traveling wave solutions involving parameter of the generalized FitzHugh-Nagumo equation.

\section{References}

[1] A. K. Ray and J. K. Bhattacharjee, "Standing and Travelling Waves in the Shallow-Water Circular Hydraulic Jump," Physics Letters A, Vol. 371, No. 3, 2007, pp. 241-248.

[2] I. E. Inan and D. Kaya, "Exact Solutions of Some Nonlinear Partial Differential Equations,” Physica A, Vol. 381, No. 1, 2007, pp. 104-115. doi:10.1016/j.physa.2007.04.011

[3] V. A. Osipov, "An Exact Solution for a Fractional Disclination Vortex,” Physics Letters A, Vol. 193, No. 1, 
1994, pp. 97-101. doi:10.1016/0375-9601(94)00664-4

[4] P. M. Jordan and A. A. Puri, "Note on Traveling Wave Solutions for a Class of Nonlinear Viscoelastic Media," Physics Letters A, Vol. 335, No. 2-3, 2005, pp. 150-156. doi:10.1016/j.physleta.2004.11.058

[5] Z. Y. Yan, "Generalized Method and Its Application in the Higher-Order Nonlinear Schrodinger Equation in Nonlinear Optical Fibres," Chaos Solitons and Fractals, Vol. 16, No. 5, 2003, pp. 759-766. doi:10.1016/S0960-0779(02)00435-6

[6] K. Nakkeeran, "Optical Solitons in Erbium Doped Fibers with Higher Order Effects,” Physics Letters A, Vol. 275, No. 5-6, 2000, pp. 415-418. doi:10.1016/S0375-9601(00)00600-9

[7] R. Hirota, "Exact Solution of the Korteweg-De Vries Equation for Multiple Collisions of Solitons,” Physical Review Letters, Vol. 27, No. 18, 1971, pp. 1192-1194. doi:10.1103/PhysRevLett.27.1192

[8] M. R. Miurs, "Backlund Transformation,” Springer, Berlin, 1978.

[9] J. Weiss, M. Tabor and G. Carnevale, "The Painlevé Property for Partial Differential Equations,” Journal of Mathematical Physics, Vol. 24, No. 3, 1983, pp. 522-526. doi:10.1063/1.525721

[10] C. T. Yan, "A Simple Transformation for Nonlinear Waves,” Physics Letters A, Vol. 224, No. 1-2, 1996, pp. 77-84. doi:10.1016/S0375-9601(96)00770-0

[11] M. L. Wang, "Exact Solution for a Compound KdvBurgers Equations,” Physics Letters A, Vol. 213, No. 5-6, 1996, pp. 279-287. doi:10.1016/0375-9601(96)00103-X

[12] M. El-Shahed, “Application of He’s Homotopy Perturbation Method to Volterra's Integro-Differential Equation,” International Journal of Nonlinear Sciences and Numerical Simulation, Vol. 6, No. 2, 2005, pp. 163-168.

[13] J. H. He, "Homotopy Perturbation Method for Bifurcation of Nonlinear Problems," International Journal of Nonlinear Sciences and Numerical Simulation, Vol. 6, No. 2, 2005, pp. 207-208.

[14] J. H. He, “Application of Homotopy Perturbation Method to Nonlinear Wave Equations," Chaos Solitons and Fractals, Vol. 26, No. 3, 2005, pp. 695-700. doi:10.1016/j.chaos.2005.03.006

[15] J. H. He, "Variational Iteration Method-A Kind of Nonlinear Analytical Technique: Some Examples," International Journal of Non-Linear Mechanics, Vol. 34, No. 4, 1999, pp. 699-708. doi:10.1016/S0020-7462(98)00048-1

[16] J. H. He, "Variational Iteration Method for Autonomous Ordinary Differential Systems,” Applied Mathematics and Computation, Vol. 114, No. 2-3, 2000, pp. 115-123. doi:10.1016/S0096-3003(99)00104-6

[17] L. Xu, J. H. He and A. M. Wazwaz, "Variational Iteration Method-Reality, Potential, and Challenges,” Journal of Computational and Applied Mathematics, Vol. 207, No. 1, 2007, pp. 1-2. doi:10.1016/j.cam.2006.07.021

[18] E. Yusufoglu, "Variational Iteration Method for Construction of Some Compact and Noncompact Structures of Klein-Gordon Equations,” International Journal of
Nonlinear Sciences and Numerical Simulation, Vol. 8, No. 2, 2007, pp. 153-158.

[19] J. H. He, "Some Asymptotic Methods for Strongly Nonlinear Equations," International Journal of Modern Physics B, Vol. 20, No. 10, 2006, pp. 1141-1199. doi:10.1142/S0217979206033796

[20] J. H. He, "Non-Perturbative Methods for Strongly Nonlinear Problems," Dissertation, de-Verlag im Internet GmbH, Berlin, 2006.

[21] T. A. Abassy, M. A. El-Tawil and H. K. Saleh, "The Solution of KdV and MkdV Equations Using Adomian Pade Approximation," International Journal of Nonlinear Sciences and Numerical Simulation, Vol. 5, No. 4, 2004, pp. 327-340.

[22] W. Malfliet, "Solitary Wave Solutions of Nonlinear Wave Equations," American Journal of Physics, Vol. 60, No. 7, 1992, pp. 650-654. doi:10.1119/1.17120

[23] E. G. Fan, "Extended Tanh-Function Method and Its Applications to Nonlinear Equations," Physics Letters A, Vol. 227, No. 4-5, 2000, pp. 212-218. doi:10.1016/S0375-9601(00)00725-8

[24] Z. S. Lü and H. Q. Zhang, “On a New Modified Extended Tanh-Function Method," Communications in Theoretical Physics, Vol. 39, No. 4, 2003, pp. 405-408.

[25] S. Zhang and T. C. Xia, "Symbolic Computation and New Families of Exact Non-Travelling Wave Solutions of $(3+1)$-Dimensional Kadomstev-Petviashvili Equation," Applied Mathematics and Computation, Vol. 181, No. 1, 2006, pp. 319-331. doi:10.1016/j.amc.2006.01.033

[26] S. Zhang, "Symbolic Computation and New Families of Exact Non-Travelling Wave Solutions of $(2+1)$-Dimensional Konopelchenko-Dubrovsky Equations," Chaos Solitons and Fractals, Vol. 31, No. 4, 2007, pp. 951-959. doi:10.1016/j.chaos.2005.10.064

[27] E. G. Fan, “Travelling Wave Solutions in Terms of Special Functions for Nonlinear Coupled Evolution Systems," Physics Letters A, Vol. 300, No. 2-3, 2002, pp. 243-249. doi:10.1016/S0375-9601(02)00776-4

[28] E. Yomba, "The Modified Extended Fan Sub-Equation Method and Its Application to the $(2+1)$-Dimensional Broer-Kaup-Kupershmidt Equation,” Chaos Solitons and Fractals, Vol. 27, No. 1, 2006, pp. 187-196. doi:10.1016/j.chaos.2005.03.021

[29] S. Zhang and T. C. Xia, “A Further Improved Extended Fan Sub-Equation Method and Its Application to the (3+ 1)-Dimensional Kadomstev-Petviashvili Equation,” Physics Letters A, Vol. 356, No. 2, 2006, pp. 119-123. doi:10.1016/j.physleta.2006.03.027

[30] S. Zhang and T. C. Xia, "Further Improved Extended Fan Sub-equation Method and New Exact Solutions of the (2 + 1)-Dimensional Broer-Kaup-Kupershmidt Equations," Applied Mathematics and Computation, Vol. 182, No. 2, 2006, pp. 1651-1660. doi:10.1016/j.amc.2006.06.004

[31] S. K. Liu, Z. T. Fu, S. D. Liu and Q. Zhao, “Jacobi Elliptic Function Expansion Method and Periodic Wave Solutions of Nonlinear Wave Equations," Physics Letters A, Vol. 289, No. 1-2, 2001, pp. 69-74. doi:10.1016/S0375-9601(01)00580-1 
[32] Z. T. Fu, S. K. Liu, S. D. Liu and Q. Zhao, "New Jacobi Elliptic Function Expansion Method and Periodic Wave Solutions of Nonlinear Wave Equations," Physics Letters A, Vol. 290, No. 1-2, 2001, pp. 72-76. doi:10.1016/S0375-9601(01)00644-2

[33] E. J. Parkes, B. R. Duffy and P. C. Abbott, "The Jacobi Elliptic-Function Method for Finding Periodic-Wave Solutions to Nonlinear Evolution Equations," Physics Letters A, Vol. 295, No. 5-6, 2002, pp. 280-286. doi:10.1016/S0375-9601(02)00180-9

[34] Y. B. Zhou, M. L. Wang and Y. M. Wang, "Periodic Wave Solutions to A Coupled KdV Equations with Variable Coefficients," Physics Letters A, Vol. 308, No. 1, 2003, pp. 31-36. doi:10.1016/S0375-9601(02)01775-9

[35] D. S. Wang and H. Q. Zhang, "Further Improved F-Expansion Method and New Exact Solutions of Konopelchenko-Dubrovsky Equation," Chaos Solitons and Fractals, Vol. 25, No. 3, 2005, pp. 601-610. doi:10.1016/j.chaos.2004.11.026

[36] S. Zhang and T. C. Xia, “A Generalized F-Expansion Method and New Exact Solutions of KonopelchenkoDubrovsky Equations,” Applied Mathematics and Computation, Vol. 183, No. 2, 2006, pp. 1190-1200. doi:10.1016/j.amc.2006.06.043

[37] Sirendaoreji and J. Sun, “Auxiliary Equation Method for Solving Nonlinear Partial Differential Equations,” Physics Letters A, Vol. 309, No. 5-6, 2003, pp. 387-396. doi:10.1016/S0375-9601(03)00196-8
[38] S. Zhang and T. C. Xia, "A Generalized Auxiliary Equation Method and Its Application to $(2+1)$-Dimensional Asymmetric Nizhnik-Novikov-Vesselov Equations," Journal of Physics A: Mathematical and Theoretical, Vol. 40, No. 2, 2007, pp. 227-248. doi:10.1088/1751-8113/40/2/003

[39] S. Zhang and T. C. Xia, “A Generalized New Auxiliary Equation Method and Its Applications to Nonlinear Partial Differential Equations,” Physics Letters A, Vol. 363, No. 5-6, 2007, pp. 356-360. doi:10.1016/j.physleta.2006.11.035

[40] S. Zhang, "A Generalized Auxiliary Equation Method and Its Application to the (2+1)-Dimensional KdV Equations," Applied Mathematics and Computation, Vol. 188, No. 1, 2007, pp. 1-6. doi:10.1016/j.amc.2006.09.068

[41] M. L. Wang, X. Z. Li and J. L. Zhang, "The $\left(\frac{G^{\prime}}{G}\right)$-Expansion Method and Travelling Wave Solutions of Nonlinear Evolution Equations in Mathematical Physics," Physics Letters A, Vol. 372, No. 4, 2008, pp. 417-423. doi:10.1016/j.physleta.2007.07.051

[42] S. Zhang, J. L. Tong and W. Wang, "A Generalized $\left(\frac{G^{\prime}}{G}\right)$-Expansion Method for the MkdV Equation with Variable Coefficients,” Physics Letters A, Vol. 372, No. 13, 2008, pp. 2254-2257. doi:10.1016/j.physleta.2007.11.026 